\title{
Four Central Points About Coevolution
}

\author{
John N. Thompson
}

Published online: 21 January 2010

(C) The Author(s) 2010. This article is published with open access at Springerlink.com

\begin{abstract}
Much of evolution is about the coevolution of species with each other. In recent years, we have learned that coevolution is much more pervasive, dynamic, and relentless than we previously thought. There are four central points about coevolution that we should teach the next generation of students to help them understand the importance of the coevolutionary process in shaping the web of life. (1) Complex organisms require coevolved interactions to survive and reproduce. (2) Species-rich ecosystems are built on a base of coevolved interactions. (3) Coevolution takes multiple forms and generates a diversity of ecological outcomes. (4) Interactions coevolve as constantly changing geographic mosaics.
\end{abstract}

Keywords Coevolution · Competition .

Evolutionary arms race $\cdot$ Mutualism $\cdot$ Symbiosis

Much of evolution is coevolution - of parasites and hosts, predators and prey, competitors, and mutualists. Interacting species impose selection on each other, continually reshaping each other's traits and life histories. This process of reciprocal evolutionary change driven by natural selection has molded the web of life, resulting in a world that not only has millions of species but also tens of millions of interactions among those species. Coevolution shapes the internet of life.

J. N. Thompson $(\bowtie)$

Department of Ecology and Evolutionary Biology,

University of California, Santa Cruz,

Santa Cruz, CA 95064, USA

e-mail: thompson@biology.ucsc.edu
It seems odd, then, that until recently the coevolutionary process has received so little discussion in most biology textbooks, although there are some exceptions (e.g., Futuyma 2009; Zimmer 2009). We could give a truer picture of the evolution of the web of life just by noting four points when trying to explain to students, and even to other scientists and science teachers, what coevolution is about and why it matters. I have written about these four central points elsewhere (Thompson 2009), and my goal here is to elaborate why they are central to our understanding of the web of life.

\section{Complex organisms require coevolved interactions to survive and reproduce}

Our own genetic code is incomplete, as is that of most other species. All complex organisms are the result of coevolution between two or more other species, and there is increasing evidence that even the simplest single-celled organisms may have diversified directly as a result of coevolution with other species (Lake 2009). Throughout the history of life, natural selection has time and again favored individuals that exploit the whole genomes of other species to survive and reproduce (Table 1).

Mitochondria are the clearest example. Like all complex organisms, we rely upon mitochondria for energy production in our cells. There is strong evidence that mitochondria evolved from ancient bacteria that coevolved with early eukaryotic organisms (Cavalier-Smith 2009). The relationship between mitochondria and their hosts eventually became so intimate that we no longer even think of these bacteria as species. Instead, we call them organelles. Plants are genetically even more complex, because most require three coevolved genomes: a nuclear genome, a mitochon- 
Table 1 Some coevolved interactions that have been crucial to the diversification of the web of life

\begin{tabular}{llll}
\hline Example & Taxon 1 & Taxon 2 & Ecological implications \\
\hline $\begin{array}{l}\text { Survival and growth } \\
\text { Mitochondria }\end{array}$ & Eukaryotes & Bacteria & Cellular energy \\
Chloroplasts & Eukaryotes & Cyanobacteria & Photosynthesis \\
Marine reefs & Corals & Dinoflagellates & Photosynthesis \\
Lichens & Fungi & Green algae/cyanobacteria & Nutrition \\
Mycorrhizae & Plants & Fungi & Plant nutrition \\
Rhizobia & Plants & Bacteria & Nitrogen fixation in soil \\
Gut symbionts & Animals & Bacteria & Digestion in animals \\
Gut symbionts & Termites & Protozoa, bacteria & Ability to digest cellulose \\
Fungus gardens & Ants & Fungi & Agriculture by ants \\
Chemosymbiosis & Bacteria & Invertebrates & Colonization of deep sea vents \\
Competition & Many taxa & Many taxa & Ecological diversification \\
Reproduction & & & Sexual reproduction in plants \\
Pollination & Plants & Animals & Sexual reproduction in plants \\
Seed dispersal & Plants & Animals & Selection for sexual reproduction \\
Parasitism & Hosts & Parasites &
\end{tabular}

Not every pair of species in these interactions is always undergoing coevolution, but the interactions have originated through the coevolutionary process and then often diversified into a wide range of ecological outcomes. See Thompson (1994; 2005) for further explanation of individual examples and references to particular studies

drial genome, and a chloroplast genome that originated from cyanobacteria and now carries out photosynthesis in plants (Archibald 2009). Hence, plants are, at minimum, a multispecies coevolved partnership among three former species.

Few plants and animals stop there in their reliance on coevolved interactions. Most require multiple other genomes to survive and reproduce in the wild. The great majority of plant species would quickly go extinct in nature without the mycorrhizal fungi attached to their roots that aid in nutrient capture or without the pollinators that aid in their reproduction. Animals generally rely on an assortment of gut symbionts to aid in nutrition, and the complexity of those symbioses is only starting to be unraveled (van Borm et al. 2002; Douglas 2009; Noda et al. 2009; Round and Mazmanian 2009).

Natural selection, then, does not adapt species in some general way to their "local environment." Much of evolution is driven by natural selection directly favoring those individuals best able to co-opt the entire genetic machinery of other species. Plant reproduction is the most graphic case, because the very act of mating in plants often requires other species. By manipulating animals to serve as pollinators, plants have outsourced the movement of their gametes from one individual to another. In general, life on earth would have been fundamentally different without the ability of organisms to evolve by co-opting other species and forming coevolved partnerships.

\section{Species-rich ecosystems are built on a base of coevolved interactions}

When we describe the organization of biological communities, we often focus on competition and predation and perhaps mention parasitism and a few instances of mutualism like pollination. This creates the impression that biological communities are mostly about antagonistic relationships among species, with some other interactions added on to the edges. That view fails completely to capture one of the most fundamental facts of biology: intimate coevolved interactions, often mutualistic, form the base of all species-rich ecosystems. Without these coevolved interactions, highly diverse ecosystems would collapse immediately.

In terrestrial communities, much of the organization happens below ground and near the surface through coevolved symbioses. Lichens, which are coevolved interactions between fungi and algae, form an important part of primary succession in many ecosystems. Mycorrhizae, which are coevolved interactions between fungi and plants, form on the roots of most plants and affect plant nutrition and growth. Rhizobia, which are coevolved interactions between bacteria and plants, form on the roots of legumes and some other plants and fix nitrogen that then becomes available for plant growth. Although these interactions are often mutualistic, they can also be antagonistic under some environmental conditions, resulting in ongoing, complex 
coevolutionary relationships in these species at the base of the food web (Piculell et al. 2008).

Similarly, coevolved interactions form the base of oceanic food webs. Much of the diversity of life in the oceans is clustered around reef-building corals that harbor mutualistic dinoflagellates. In fact, the increasing environmental problem of coral bleaching in many coral reefs is the result of the loss of these dinoflagellates and the subsequent death of the corals (Weis 2008). In the surrounding open ocean, the planktonic communities at the base of the food web rely on coevolved interactions among tiny species that have only recently been studied in detail. Cyanobacteria in the genus Prochlorococcus are one of the major photosynthetic organisms in the open ocean, and by some estimates they are the most abundant photosynthetic organisms on earth. The genes for photosynthesis in these species show evidence of repeated transfer back and forth between the cyanobacterial genome and the genomes of the viruses with which they coevolve (Lindell et al. 2004, 2007). The result is a complex distribution of genes for photosynthesis among these coevolving, and ecologically important, hosts and viruses (Sharon et al. 2009).

Farther down in the depths of the ocean where sunlight never reaches, other communities are built directly on yet other coevolved interactions. As deep-sea vents exude sulfur and methane, these chemicals are converted to usable energy by specialized microbial species that live in symbiotic association with mussels, clams, shrimps, gastropods, polychaetes, and tubeworms (Nakagawa and Takai 2008). These taxa form the base of the web of life surrounding these vents.

Almost everywhere on earth, then, coevolved interactions have made it possible for organisms to exploit new environments, thereby fueling the further diversification of life. The result is not a Gaia-like world functioning as a self-sustaining unit. Rather, it is a wildly dynamic world of constantly coevolving mutualistic and antagonistic interactions. The coevolutionary process has fueled such a diversity of life and lifestyles that, at least so far, some species have made it through each of the periods of mass extinction that have occurred during our earth's history.

\section{Coevolution takes multiple forms and generates a diversity of ecological outcomes}

The diversity of lifestyles that we see today in all ecological communities has come from the diversity of the coevolutionary process itself. There is no single form or direction of coevolutionary change, just as there is no single form or direction of evolutionary change. Through studies of a wide range of interactions in recent decades, we now know that the coevolutionary process has multiple identifiable forms
(Table 2). The most extreme form of antagonistic coevolution is coevolutionary escalation, which generates "arms races" between prey and predators. Selection favors individuals that devote a higher proportion of energy to defense or counterdefense than other individuals. The coevolutionary process continues until stopped by tradeoffs imposed on populations by other requirements, such as the need to compete with other species, find mates, or rear offspring. How natural selection balances all these conflicting demands varies among environments, leading sometimes to complex geographic patterns in levels of defense and counter-defense in interacting species (Hanifin et al. 2008). Over longer geological time scales, these coevolutionary arms races have fueled some of the diversification of life (Ehrlich and Raven 1964; Farrell 1998; Becerra et al. 2009; McKenna et al. 2009; Segraves 2010).

Antagonistic interactions also coevolve in more subtle ways. Parasites and hosts often coevolve through selection that favors rare, rather than more highly defended, genetic forms (Lively 2010). Local parasites are often not adapted to genetically rare forms of their local host population. Consequently, these rare host forms have higher survival or reproduction than genetically more common hosts. As a rare host form increases in frequency in the population, natural selection starts to favor genetic forms of the parasite able to attack them. Over time, selection fluctuates on the host and parasite populations, favoring first one and then another genetic form of the host and the parasite. Eventually, the host and parasite populations accumulate multiple genetic forms (i.e., they become increasingly polymorphic). The evolution of sexual reproduction itself may be a result of this common form of coevolutionary selection (Lively 2010). Unlike in asexual females, a sexual female produces offspring that are genetically different from her, and one or more of those offspring may be a genetically rare form that is relatively immune to attack by local parasites.

A similar process of fluctuating selection, called coevolutionary alternation, may occur when multiple prey (or hosts) coevolve with one or more predators (or parasites) (Davies and Brooke 1989; Thompson 2005; Nuismer and Thompson 2006). In this form of multispecific coevolution, a predator species varies genetically over time in its preference for particular prey species, and prey species fluctuate genetically over time in their levels of defense. During this process, natural selection favors predators that preferentially attack the local prey species with the currently lowest levels of defense. That favors increased defenses in the prey populations that are currently under heavy attack, causing selection then to shift and favor genetic forms of the predator that preferentially attack other host species with lower defenses. Over long periods of time, genetically-based preferences for hosts continue to shift within each predator population, and the levels of 
Table 2 The various ways in which local populations of species coevolve

\begin{tabular}{lll}
\hline Form of interaction & Form of coevolution & Primary form of selection $^{\text {a }}$ \\
\hline Mutualism & Coevolving complementarity & Directional; stabilizing \\
Mutualism & Coevolutionary convergence & Directional; stabilizing \\
Trophic antagonistic & Coevolutionary escalation & Directional \\
Trophic antagonistic & Coevolving polymorphisms & Fluctuating \\
Trophic antagonistic & Coevolutionary alternation & Fluctuating \\
Competitive & Coevolutionary displacement & Directional \\
Trophic antagonistic & Attenuated antagonism & Directional \\
\hline
\end{tabular}

Modified from Thompson (2005)

${ }^{a}$ Multiple forms of selection act on most of these forms of coevolution, but only the primary forms are shown here. See Thompson (2005) for further discussion

defense in each prey species repeatedly fluctuate up and down. This form of selection has the potential to create coevolving groups of predators and prey, with different combinations of species interacting at different moments in evolutionary history. This form of coevolution has been shown in mathematical models to be feasible, and it has been suggested as an explanation for the dynamics of some coevolving interactions, but it has not yet been fully demonstrated for any coevolving interaction (Davies and Brooke 1989; Thompson 2005; Nuismer and Thompson 2006).

Coevolution among competitors differs from other forms of antagonistic interaction, because the interaction is detrimental to the fitness of both species. Coevolutionary selection on competitors therefore tends to favor individuals with traits that make them less likely to compete with other species using the same limited resources. The traits of these competing species become displaced in ecosystems where they compete. Hence, clues to coevolution through character displacement can often be found by studying multiple populations of potentially competing species. Initial evidence for competition comes from observations indicating that the traits of species differ more in regions where they co-occur than in regions in which only one of the species occurs, or the traits differ geographically in predictable ways depending on which combinations of species co-occur (Schluter 2000; Losos 2009; Schluter 2010). Those differences may involve divergence in traits (e.g., body size), the habitats used by the competing species, or the times of year that the species use a particular habitat, and they may also involve multiple forms of selection other than competition (Pfennig and Pfennig 2009).

Finally, some antagonistic interactions may even coevolve toward decreased antagonism under some ecological conditions. Decades ago, it was commonly assumed that parasites and hosts would gradually coevolve toward lower levels of antagonism, but we now know that occurs only under some conditions. If a parasite can propagate only by being passed directly from a host female to her offspring, then natural selection is likely to favor less virulent forms of the parasite - that is, reduced antagonism. The reason is that less virulent parasites do not kill the host before she is able to reproduce, allowing the parasite to transmit her offspring to the host's offspring. If, however, parasites propagate through infectious spread (e.g., by one host sneezing onto another), then natural selection often favors virulent forms of the parasite. These parasites make many copies of themselves at the host's expense and then quickly spread to new hosts. Hence, parasites can evolve to become either more virulent or less virulent, depending partially on how they get from one host to the next.

It is, though, not quite that simple. Research on the evolution of virulence has shown that many factors other than how parasites are transmitted can affect the evolution of virulence. These include the rates of co-infection by other parasites, the average time between infection and transmission to the next host, and how selection acts on the host (Alizon et al. 2009). We now understand, though, much better than before why the degree of virulence is so variable among parasites as they coevolve with their hosts.

At the other extreme from antagonistic interactions, mutualistic species increase the Darwinian fitness of each other, but they do so by mutual exploitation. In extreme forms of mutualism, a pair of interacting species, such as a gut symbiont and its host, might coevolve to be so complementary that they literally cannot survive without each other. Such extreme complementarity occurs commonly in mutualistic interactions in which symbionts live in the host and are passed directly to the offspring of the host (Moran et al. 2008).

But coevolution does not lead to extreme reciprocal specialization in all mutualistic interactions. It is uncommon in mutualisms between free-living species such as those between plants and their pollinators and seeddispersal agents. That lack of extreme specialization appears to be a result of the coevolutionary process itself (Thompson 2005). It may seem paradoxical that coevolution would actually favor larger groups of interacting 
species rather than highly specialized pairs of species, but we are now beginning to understand how this process of multispecific coevolution may work.

The reason is the coevolutionary vortex. Mutualisms among free-living species tend to draws in other species over time, creating a tangled web of interactions. As coevolution favors complementarity between partners (e.g., flowers and hummingbirds), it simultaneously favors other related or unrelated species that evolve to exploit the interaction by converging on those same traits. In one wellstudied community in Costa Rica 65 hawkmoth species interact with 31 plant species from various plant families that have converged on floral traits adapted to pollination by hawkmoths (Haber and Frankie 1989). The scientific problem of how coevolution shapes larger webs of interacting species is one of the most active areas of current coevolutionary research, and there is still a great deal to learn (Bascompte et al. 2006; Thompson 2006; Bascompte and Jordano 2007; Guimarães et al. 2007; Jordano 2010).

\section{Interactions coevolve as constantly changing geographic mosaics}

Perhaps one of the biggest changes to have occurred in recent years in our understanding of coevolution is that we now know that it is a relentless ecological process and not something rare and observable only over long periods of geologic time. We can see evidence of ongoing coevolution by studying the same interaction in multiple places. Each group of species interacting in a local community is a potential mini-coevolutionary experiment. Each local population of those species is often genetically distinct from other populations of the same species. And each of those populations interacts in unique ways with other species, because each environment imposes unique selection pressures.

Consequently, interacting species often coevolve in some parts of their geographic ranges, creating coevolutionary hotspots where selection is reciprocal, but not in other parts, creating coevolutionary coldspots where reciprocal selection does not occur (Fig. 1). A parasite might impose strong selection on its host population in some environments, but elsewhere live as a commensal on that same host species. Even if an interaction coevolves everywhere, it may do so in different ways in different ecosystems. These geographic selection mosaics and coevolutionary hotspots and coldspots are, in turn, continually remolded as new traits appear and disappear among populations through mutation, gene flow, random genetic drift, and the dynamics of metapopulations (Thompson 1994; 2005). The result is a constantly changing geographic mosaic of coevolving interactions, and the geographic mosaic theory of coevolution has

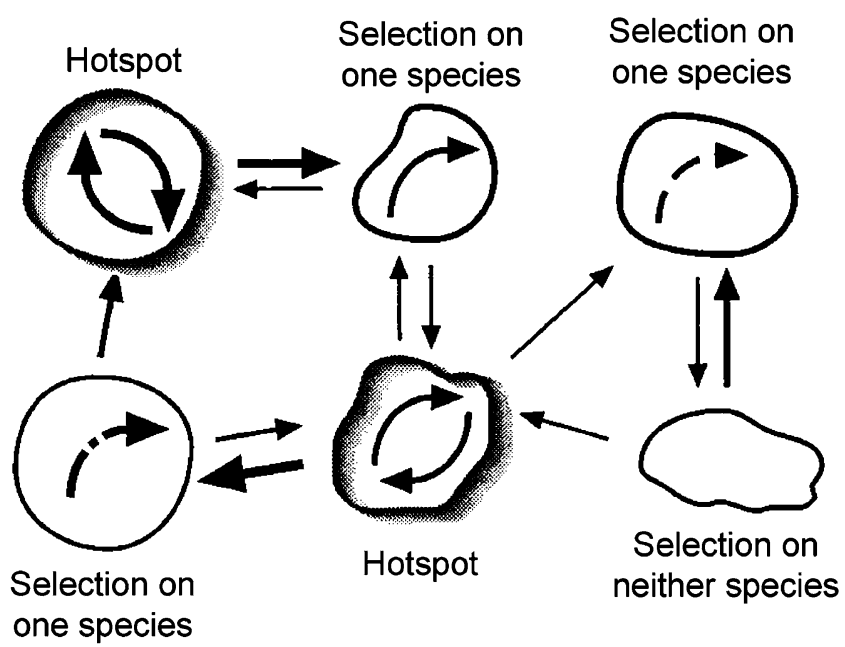

Fig. 1 Diagrammatic representation of some of the major components of the geographic mosaic of coevolution between a pair of species. Interactions within local communities are shown as arrows within circles and indicate selection acting on either one or both species. Different arrow angles represent differences among communities in how selection acts on the species. Differences in arrow thickness and lines represent differences in the strength or form of natural selection. Arrows between communities indicate gene flow, with thicker arrows representing more gene flow. In this diagram, coevolutionary hotspots (i.e., communities in which selection is reciprocal on the interacting species) occur within a broader matrix of coevolutionary coldspots (i.e., communities in which selection either does not act on the interacting species or is not reciprocal). Modified from Thompson (2005)

provided a framework for studying how these processes collectively shape the adaptation and diversification of interactions among species.

We now have excellent studies of these mosaics. For example, squirrels have coevolved with conifers in many regions of North America and Eurasia, but where the squirrels do not occur these conifers have coevolved with crossbills. The traits of the conifers and the crossbills show just the kinds of geographic differences you would expect to find in regions with squirrels as compared with regions without squirrels (Edelaar and Benkman 2006; Siepelski and Benkman 2007; Parchman and Benkman 2008; Benkman and Parchman 2009; Benkman 2010). Toxic newts and the garter snakes that eat them differ geographically in western North America in the level of chemical defenses in the newts and the ability of the snakes to detoxify those chemical defenses (Brodie et al. 2002; Brodie et al. 2005; Hanifin et al. 2008). Wild parsnips and parsnip webworms, introduced to North America from Europe only within the past few hundred years, have coevolved into a geographic mosaic in the mix of chemical defenses deployed by the plants against these insects and the detoxification enzymes in the insects that counteract these defense chemicals (Berenbaum and Zangerl 1998; Zangerl and Berenbaum 2003). Hummingbirds and Heliconia flowers differ among islands in the Caribbean in ways that reflect differences in 
their ecological interactions in different ecosystems (Temeles and Kress 2003).

Examples like these of coevolutionary mosaics continue to be found each year, now that researchers are asking how these mini-coevolutionary "experiments" have been shaped by selection in different ways in different places (Burdon et al. 2002; Thrall et al. 2002; Laine 2005; Thompson and Fernandez 2006; Nash et al. 2008; Toju 2008; Anderson and Johnson 2009; King et al. 2009; Johnson and Anderson 2010; Medel 2010). Meanwhile, multiple researchers are generating their own geographic mosaics by constructing complex microcosms in the laboratory (e.g., connected flasks). These studies are demonstrating, over just a few weeks and months, rapid evolution of geographic mosaics in coevolving bacteria and phage (Forde et al. 2007; LopezPascua and Buckling 2008; Vogwill et al. 2009; Brockhurst 2010). Through all these studies we are developing a much better understanding of how species continue to coevolve in different ways across continually changing environments.

\section{Final Points}

This is an exciting time in coevolutionary biology as new discoveries occur each year. A few decades ago, we did not know enough about the coevolutionary process to make the four central points I have emphasized here. We can do so now because coevolutionary biology has advanced quickly in recent years as researchers have adopted new genetic, ecological, geographical, mathematical, and statistical approaches. We now know that coevolution is a much more pervasive, dynamic, and ongoing process than we ever suspected.

By teaching these four central points about coevolution to the next generation of students and researchers, we would go a long way toward helping them understand the importance of coevolution in the organization of ecosystems and the diversification of life. We would also be preparing them to understand how the coevolutionary process itself may be changing as we are rapidly altering all the major ecosystems on earth. That knowledge would help the next generation of biologists formulate ways to preserve the conditions needed to maintain the coevolving web of life.

Acknowledgments I thank Niles Eldredge for helpful comments on the manuscript, Rodrigo Medel for discussions throughout the development of this special volume, and the authors of this special issue who have contributed so much to the development of our understanding of the coevolutionary process. This work was supported by NSF grant DEB-0839853.

Open Access This article is distributed under the terms of the Creative Commons Attribution Noncommercial License which permits any noncommercial use, distribution, and reproduction in any medium, provided the original author(s) and source are credited.

\section{References}

Alizon S, Hurford A, Mideo N, Van Baalen M. Virulence evolution and the trade-off hypothesis: history, current state of affairs and the future. J Evol Biol. 2009;22:245-59.

Anderson B, Johnson SG. Geographical covariation and local convergence of flower depth in a guild of fly-pollinated plants. New Phytologist. 2009;182:533-40.

Archibald JM. The origin and spread of eukaryotic photosynthesis: evolving views in light of genomics. Bot Mar. 2009;52:95-103.

Bascompte J, Jordano P. Plant-animal mutualistic networks: the architecture of biodiversity. Ann Rev Ecol, Evolut, Systs. 2007;38:567-93.

Bascompte J, Jordano P, Olesen JM. Asymmetric coevolutionary networks facilitate biodiversity maintenance. Science. 2006;312: 431-3.

Becerra JX, Noge K, Venable DL. Macroevolutionary chemical escalation in an ancient plant-herbivore arms race. Proc Natl Acad Sci USA. 2009;106:18062-6.

Benkman CW. Diversifying coevolution between crossbills and conifers. Evolution: Education and Outreach. 2010. doi:10.1007/ s12052-009-0190-8.

Benkman CW, Parchman TL. Coevolution between crossbills and black pine: the importance of competitors, forest area and resource stability. J Evol Biol. 2009;22:942-53.

Berenbaum MR, Zangerl AR. Chemical phenotype matching between a plant and its insect herbivore. Proc Natl Acad Sci USA. 1998;95:13743-8.

Brockhurst M. Using microbial microcosms to study host-parasite coevoluiton. Evolution: Education and Outreach. 2010. doi:10.1007/ s12052-009-0188-2.

Brodie Jr ED, Ridenhour BJ, Brodie III ED. The evolutionary response of predators to dangerous prey: hotspots and coldspots in the geographic mosaic of coevolution between newts and snakes. Evolution. 2002;56:2067-82.

Brodie EDI et al. Parallel arms races between garter snakes and newts involving tetrodotoxin as the phenotypic interface of coevolution. J Chem Ecol. 2005;31:343-56.

Burdon JJ, Thrall PH, Lawrence GJ. Coevolutionary patterns in the Linum marginale-Melampsora lini association at a continental scale. Can J Bot. 2002;80:288-96.

Cavalier-Smith T. Predation and eukaryote cell origins: a coevolutionary perspective. Int J Biochem Cell Biol. 2009;41:307-22.

Davies NB, Brooke MdL. An experimental study of co-evolution between the cuckoo, Cuculus canorus, and its hosts. II. Host egg markings, chick discrimination and general discussion. J Anim Ecol. 1989;58:225-36.

Douglas, AE. The microbial dimension in insect nutritional ecology. Funct Ecol. 2009;23:38-47.

Edelaar P, Benkman CW. Replicated population divergence caused by localized coevolution? A test of three hypotheses in the red crossbill-lodgepole pine system. J Evol Biol. 2006;19:1652-9.

Ehrlich PR, Raven PH. Butterflies and plants: a study in coevolution. Evolution. 1964;18:586-608.

Farrell BD. "Inordinate fondness" explained: why are there so many beetles? Science. 1998;281:555-9.

Forde SE, Thompson JN, Bohannan BJM. Gene flow reverses an adaptive cline in a coevolving host-parasitoid interaction. Am Nat. 2007;169:794-801.

Futuyma DJ. Evolution. 2nd ed. Sunderland, Massachusetts: Sinauer Associates; 2009.

Guimarães Jr PR, Rico-Gray V, Oliveira PS, Izzo TJ, dos Reis SF, Thompson JN. Interaction intimacy affects structure and coevolutionary dynamics in mutualistic networks. Curr Biol. 2007;17:1797-803. 
Haber WA, Frankie GW. A tropical hawkmoth community: Costa Rican dry forest Sphingidae. Biotropica. 1989;21:155-72.

Hanifin CT, Brodie EDJ, Brodie EDI. Phenotypic mismatches reveal escape from arms-race coevolution. PLOS Biology. 2008;6:e60.

Johnson SD, Anderson R. Coevolution between food-rewarding flowers and their pollinators. Evolution: Education and Outreach. 2010. doi:10.1007/s12052-009-0192-6.

Jordano P. Coevolution in multi-specific interactions among free-living species. Evolution: Education and Outreach. 2010. doi:10.1007/ s12052-009-0197-1.

King KC, Delph LF, Jokela J, Lively CM. The geographic mosaic of the Red Queen: more sex in coevolutionary hotspots. Current Biology. 2009;19:1438-1441.

Laine A-L. Spatial scale of local adaptation in a plant-pathogen metapopulation. J Evol Biol. 2005;18:930-8.

Lake JA. Evidence for an early prokaryotic endosymbiosis. Nature. 2009;460:967-71.

Lindell D, Jaffee JD, Coleman ML, Futschik ME, Axmann IM, Rector T, et al. Genome-wide expression dynamics of a marine virus and host reveal features of co-evolution. Nature. 2007;449:8386.

Lindell D, Sullivan MB, Johnson ZI, Tolonen AC, Rohwer F, Chisholm SW. Transfer of photosynthesis genes to and from Prochlorococcus viruses. Proceedings of the National Academy of Sciences USA. 2004;101:11013-11018.

Lively CM. Antagonistic coevolution and sex. Evolution: Education and Outreach. 2010. doi:10.1007/s12052-009-0196-2.

Lopez-Pascua LDC, Buckling A. Increasing productivity accelerates host-parasite coevolution. J Evol Biol. 2008;21:853-60.

Losos JB. Lizards in an evolutionary tree: ecology and adaptive radiation of anoles. Berkeley: University of California Press; 2009.

McKenna D, Sequeira A, Marvaldi A. Temporal lags and overlap in the diversification of weevils and flowering plants. Proc Natl Acad Sci U S A. 2009;106:7083-8.

Medel R. Arms race coevolution: the local and geographic structure of a host-parasite interaction. Evolution: Education and Outreach. 2010. doi:10.1007/s12052-009-0191-7.

Moran NA, McCutcheon JP, Nakabachi A. Genomics and evolution of heritable bacterial symbionts. Annu Rev Genet. 2008;42:165-90.

Nakagawa S, Takai K. Deep-sea vent chemoautotrophs: diversity, biochemistry and ecological significance. FEMS Microbiol Ecol. 2008;65:1-14.

Nash DR, Als TD, Maile R, Jones GR, Boomsma JJ. A mosaic of chemical coevolution in a large blue butterfly. Science. 2008;319:88-90.

Noda S, Hongoh Y, Sato T, Ohkuma M. Complex coevolutionary history of symbiotic Bacteroidales bacteria of various protists in the gut of termites. BMC Evol Biol. 2009;9:158.

Nuismer SL, Thompson JN. Coevolutionary alternation in antagonistic interactions. Evolution. 2006;60:2207-17.

Parchman TL, Benkman CW. The geographic selection mosaic for ponderosa pine and crosbills: a tale of two squirrels. Evolution. $2008 ; 62: 348-60$
Pfennig K, Pfennig D. Character displacement: ecological and reproductive responses to a common evolutionary problem. Q Rev Biol. 2009;83:253-76.

Piculell BJ, Hoeksema JD, Thompson JN. Interactions of biotic and abiotic environmental factors on an ectomycorrhizal symbiosis, and the potential for selection mosaics. BMC Biology. 2008;6:23.

Round JL, Mazmanian SK. The gut microbiota shapes intestinal immune responses during health and disease. Nat Rev Immunol. 2009;9:313-323.

Schluter D. The ecology of adaptive radiation. Oxford: Oxford University Press; 2000.

Schluter D. Resource competition and coevolution in sticklebacks. Evolution: Education and Outreach. 2010. doi:10.1007/s12052009-0204-6.

Segraves KA. Branching out with coevolutionary trees. Evolution: Education and Outreach. 2010. doi:10.1007/s12052-009-0199-z.

Sharon I et al. Photosystem I gene cassettes are present in marine virus genomes. Nature. 2009;461:258-62.

Siepelski AM, Benkman CW. Convergent patterns in the selection mosaic for two North American bird-dispersed pines. Ecol Monogr. 2007;77:203-20.

Temeles EJ, Kress WJ. Adaptation in a plant-hummingbird association. Science. 2003;300:630-3.

Thompson JN. The coevolutionary process. Chicago: University of Chicago Press; 1994.

Thompson JN. The geographic mosaic of coevolution. Chicago: University of Chicago Press; 2005.

Thompson JN. Mutualistic webs of species. Science. 2006;312:372-3.

Thompson JN. The coevolving web of life. Am Nat. 2009;173:125-40.

Thompson JN, Fernandez CC. Temporal dynamics of antagonism and mutualism in a geographically variable plant-insect interaction. Ecology. 2006;87:103-12.

Thrall $\mathrm{PH}$, Burdon JJ, Bever JD. Local adaptation in the Linum marginale-Melampsora lini host-pathogen interaction. Evolution. 2002;56:1340-51.

Toju H. Fine-scale local adaptation of weevil mouthpart length and Camellia pericarp thickness: altitudinal gradient of a putative arms race. Evolution. 2008;62:1086-102.

van Borm S, Buschinger A, Boomsma JJ, Billen J. Tetraponera ants have gut symbionts related to nitrogen-fixing root-nodule bacteria. Proceedings of the Royal Society Biological Sciences Series B. 2002;269:2023-2027.

Vogwill T, Fenton A, Buckling A, Hochberg ME, Brockhurst MA. Source populations act as coevolutionary pacemakers in experimental selection mosaics containing hotspots and coldspots. Am Nat. 2009;173:E171-6.

Weis VM. Cellular mechanisms of Cnidarian bleaching: stress causes the collapse of symbiosis. J Exp Biol. 2008;211:3059-66.

Zangerl AR, Berenbaum MR. Phenotypic matching in wild parsnip and parsnip webworms: causes and consequences. Evolution. 2003;57:806-15.

Zimmer C. The tangled bank: an introduction to evolution. Greenwood Village, Colorado: Roberts and Company; 2009. 